\title{
How Effective is the Treatment of Leprosy?
}

\author{
T. W. MEADE \\ MRC-DHSS Epidemiology and Medical Care Unit, Northwick Park Hospital, \\ Watford Road, Harrow, Middlesex HA1 3UJ, England
}

\begin{abstract}
This transcript of the 1976 Clayton Memorial Lecture sets the problem of eradicating leprosy against a similar situation affecting several other major diseases. We already have sufficient knowledge either to eradicate or radically influence the prevalence of such diseases, but they nevertheless continue with little prospect of any rapid decline. The reasons for this unbridged gap between knowledge and its effective application vary to some extent. The various factors in the "application gap" which are of relevance to leprosy are discussed and the importance of prevention as a primary objective is emphasized.
\end{abstract}

This paper aims not only to highlight some of the problems associated with the effective treatment of leprosy, but also the similarity of the difficulties experienced by health workers in more as well as less affluent countries, at a time when nearly all of them have less and less with which they are expected to do more and more. In health terms, Western society is confronted mainly by the burden of the non-communicable diseases that have largely replaced the communicable-ischaemic heart disease, stroke, lung cancer, dental caries, for example. All these conditions have one feature in common; we have the knowledge either to prevent or treat them-if not entirely, then certainly to a very substantial extent. Yet each continues and, with minor exceptions, there is no reason to believe that the situation will improve. Why is this? We can provide some reasons, mostly rather superficial-to do with the difficulties of giving up smoking and overeating, taboos about what we will or will not put in our drinking water, and so on-but basically, we do not know how to apply information already to hand. We have assumed that simply having the knowledge which would make treatment or prevention possible would be enough. People would, for example, follow the scientific argument linking cigarette smoking with lung cancer, they would accept it, stop smoking and live to see the disease disappear. After all, much of the knowledge in question was acquired soon after some of the most spectacular advances ever made in medicine; why should progress not go on being as straightforward, relatively speaking at least, as applying the knowledge that led to penicillin and its use, or to immunization against diphtheria? With hindsight, some of the reasons are obvious. Stopping smoking is no once-off affair, like having a course of penicillin or a smallpox vaccination; it involves a sustained long-term effort of will. What we are faced with at present is a largely unbridged gap between knowledge and its effective application. No-one has put this better than the late Lord Rosenheim (1968):

"If, for the next 20 years no further research were to be carried out, if there were a moratorium on research, the application of what is already known, of what has already been discovered, would result in wide-spread improvement in world health."

This paper is based on the Third Clayton Memorial Lecture, given in Liverpool on 1 December, 1976. 
That is not all he said on that occasion; the relevance of another extract is considered later. What this paper is mainly concerned with however, is the "application gap" (Lancet, 1976) in the treatment of leprosy.

For hundreds if not thousands of years, the management of leprosy was based on a mixture of fear, magic, social ostracism and a few compounds for which there were some indications, however tenuous, that they might be useful, e.g. the painful injection of chaulmoogra oil. The tendency towards self-healing in the less serious forms of the disease may have been a factor which sometimes lent support to the apparent effectiveness of some of these older remedies, and, correspondingly, hampered the pursuit of other remedies.

In the early 1940s, however, the search for anti-tuberculosis agents led to the identification of certain sulphone derivatives which were actually ineffective against tuberculosis, but were shown by Faget et al. in 1943 to be effective in leprosy. By 1952, dapsone, or DDS, had become stand ard treatment for leprosy, and has continued as the sheet-anchor ever since; it is cheap and relatively free from adverse effects. After years of standing more or less helplessly by, it must have been an exhilarating experience to be working in the leprosy field during the late 1940s and early 1950s, and, indeed, one can tell from the early literature of the sulphone era that it was. For not only was it believed that DDS at last provided effective treatment for patients with leprosy-it was assumed that this treatment would reduce the pool of infectivity in the world's endemic areas, prevent the transmission of Mycobacterium leprae to others, and thus virtually eradicate the disease. As one follows the DDS story through, the 1950s and early 1960s were still times of high hopes for both treatment and prevention; with such a long incubation period and protracted clinical course, it was hardly to be expected that things would change overnight. One of the most striking developments at this time, and partly attributable to the increasing availability of DDS, was the gradual replacement of institutional care in isolation by domiciliary management based on case-finding and DDS treatment in the community. This movement has been carried even further in some areas through attempts to discourage the view that leprosy and its treatment are special problems that differ from other health problems, and to integrate leprosy services into the local system for providing health care as a whole.

Towards the end of the 1960s, however, serious doubts about the early assumptions of the DDS era had begun to emerge. With hindsight, some of these could perhaps have been anticipated. First of all, DDS is bacteristatic-not bactericidal-and viable $M$. leprae have been demonstrated in the tissues of lepromatous patients who have been treated with DDS for many years. Secondly, the reversal reaction may lead to the very distressing situation that treatment has in fact accelerated or even caused just those deformities it is intended to prevent; this reaction is not, of course, associated only with DDS, but to the extent that DDS is the most widely used anti-leprosy agent, it is likely to be the commonest initiator of the reversal reaction. Thirdly, the parallel tuberculosis story had shown that prolonged monotherapy was an almost certain prescription for the emergence of drug resistance. On any scale, this would be a blow of the most serious kind to leprosy treatment. There are now other preparations that are effective against $M$. leprae; rifampicin, for example, is bactericidal. But none combines the qualities of widespread availability, low cost and relative non-toxicity of DDS. Resistance to DDS was initially thought to be rare, but with the passage of time it is becoming clear that this is not so. In one study (Pearson 
et al., 1975) about $2 \%$ of lepromatous patients have developed DDS resistance; as time passes, the cumulative total will almost certainly exceed this figure. Finally, the global picture today of leprosy and its consequences does not, after all, seem very different from the picture in 1952, at least to many experts. There are still millions of patients, many with serious deformities; early hopes that leprosy would now be well on its way to being a disease of the past have not been fulfilled. Yet we probably do have the means for at least some of these objectives. What then is the nature of the "application gap" in leprosy and how can we bridge it? What do we need to be able to do, between having DDS readily available on the one hand, and making sure that the right people take it in the right quantities, on the other?

First of all we have to be clear about what precisely we are trying to achieve. There is obviously no doubt that DDS is usually strikingly effective under stringently supervised clinical conditions; but only a tiny proportion of the world's leprosy patients can be treated under these rather exceptional circumstances. They live in, or, where they are rejected, alongside their own communities, and it is under these unsupervised and unsupervisable conditions that treatment will succeed or fail. Secondly, how should we measure success or failure? To the leprosy patient, what chiefly matters is whether he can live a normal and productive life within his accustomed family and community setting. In the world's endemic areas, this largely depends on the absence of significant deformity and disability, which ought increasingly to be one of our main measures of outcome. Other measures may be easier and quicker to make, and in certain circumstances are the appropriate ones-for example, the activity of skin lesions, or the number of viable bacilli. But at the end of the day, the leprosy patient's main worry is whether he has fingers or not, and whether he can use his hands and arms to earn his living; he himself is not primarily concerned with how many bacilli he is carrying, or even, usually, with the exact appearance and activity of his skin lesions. (There is very little systematic work validating the use of shorter-term microbiological and clinical endpoints as indicators of the long-term social outcome, and it would be of the greatest value to have this gap filled.) The use of new notifications, or of incidence, as an endpoint in domiciliary treatment programmes is relevant to the hypothesis that treating established cases will prevent new cases by interrupting transmission. Quite apart from the fact that figures of this kind may be difficult to interpret (Meade, 1974), it is arguable that prevention through treatment of established cases is unlikely to be achieved except under very exceptional circumstances; thus, judging community treatment by the yardstick of new notifications may be to attempt the confirmation of a hypothesis that is unjustifiably optimistic at the outset. There is, however, an even more basic reservation about the use of new notifications for assessing the impact of large-scale treatment programmes; since, by definition, these programmes aim to treat patients with established and manifest disease, they should therefore be evaluated by criteria relevant to this therapeutic objective, and not by those applicable to the objective of primary prevention. A realization that many large-scale programmes are using inappropriate measures of their success or failure, followed by a switch to more suitable indices, could be one of the most significant changes in modern approaches to leprosy.

It is interesting how closely these considerations mirror those encountered in other situations. In the case of ischaemic heart disease, for example, we have 
exactly the same problem of having to try to introduce measures which to some extent work under close supérvision-for example, dietary changes-into a largely unsupervised setting, where their acceptability and efficacy are very much more doubtful. And, secondly, there is the question of the appropriate measure of outcome; what the middle-aged man in Western society is likely to worry about is whether he is going to have a "coronary", or drop dead-not what the inside of his coronary arteries, or even his electrocardiogram, looks like. The "application gap" really does have common features across the world.

Let us take it, therefore, that while the "showcase" treatment scheme undoubtedly has its place in the study of leprosy, since this is where new ideas are tested and developed, what we must ultimately be concerned with is, first, the delivery of treatment in the much less favourable circumstances in which most leprosy patients find themselves; and secondly, with long-term social outcome.

Obviously, sheer inaccessibility, whether this is due to mountains, deserts, or the transformation of the landscape that rains may cause, has to be borne in mind. How often is it even practicable to reach patients? This is a local problem requiring local answers and a great deal of ingenuity. At the other end of the spectrum, is there a point of diminishing returns beyond which, in our anxiety to get patients to take the treatment we think they need, we actually put them off by expecting too much in the way of attendance and compliance?

LEPRA's scheme in Southern Malawi (Molesworth, 1969) has provided the opportunity to start looking at some of the questions. The data eventually available will enable outcome, measured in terms of the development of avoidance of deformities, to be assessed by various indices of regularity of DDS taking. Analyses are still in progress, but they show that many patients attend irregularly, and also suggest that regular attendance results, at best, in only marginally better outcome than irregular attendance. The precise interpretation of these results is still uncertain, but one possibility, obviously, is that DDS treatment under field conditions is not effective in terms of the onset or otherwise of deformity. Results of this kind (if confirmed) are based on the assumption that regularity of attendance, which is easy to record, is some indication of regularity of taking DDS. Unfortunately, this is very much an oversimplification. Ellard et al. (1974) developed a method for determining the regularity of DDS self-administration by out-patients, which was then applied in the setting of LEPRA's Malawi project (against a background of knowledge gained from work on tuberculosis that drug regimens that are successful in clinical trials may fail in the large-scale applied context because patients do not adhere to them). The results of this work strongly suggested that only about half of the total prescribed DDS doses had actually been taken within the day or so preceding the test. Low and Pearson (1974), reporting on a study in 89 out-patients in Ethiopia, found that only $42 \%$ of prescribed dosage had been taken in the previous 24 to $48 \mathrm{~h}$. All this applies to patients who reach an out-patients clinic in the first place, so it is only too clear that the proportion of all those with leprosy who take DDS regularly must be very low. In addition, these considerations beg the still larger unanswered question as to what is the most effective DDS regimen anyway-in terms of dosage, frequency of attendance, etc. The "application gap" is all too evident. It must, however, be re-emphasized that leprosy is not by any means the only disease with a treatment compliance problem; on the positive side, in fact, it is one of a relatively small number of conditions where serious efforts have been made to study the matter. 
There are obviously reservations and qualifications to be made about this kind of approach to evaluating leprosy treatment. The presence or absence of deformities is not the only yardstick by which we should judge the success of community treatment programmes, even though there is a strong case for regarding it as a very important one. It may be, for example, that lepromatous patients who attend regularly for treatment have less trouble from ENL and other complications than those who do not, and there are data (Quagliato et al., 1970) which strongly suggest that bacteriological and clinical relapse are largely determined by irregularity of treatment. But we ought to be prepared to accept the possibility that within the framework and constraints of large-scale community schemes, outcome is not materially affected by regularity of treatment. It could be that there is a threshold level of total DDS dosage, which would obviously largely depend on regularity of attendance, below which treatment is ineffective; that domiciliary and community programmes simply cannot achieve this level; and that it was unreasonable at the outset to expect them to do so. Apart from its possible effects on clinical outcome, the combination of low dosage and irregular treatment is almost a guarantee for the promotion of resistance to DDS, and thus another pressing reason for being concerned with the problem. If rifampicin were widely used, an additional anxiety would be that irregular treatment is a potent cause of jaundice and other adverse reactions.

In view of what we know, or suspect, about the efficacy of leprosy treatment in the community, the assumption that the DDS treatment of established cases of leprosy is also the answer to the prevention of leprosy, by interrupting the chain of transmission, is to carry optimism to unrealistic lengths. If all patients took all their treatment, if they and their potential contacts could be confined to one area, and had no contact with anyone outside it, if there were no resistance problem, and so on, then the idea could bear further thought. But all the signs are that banking on this approach to prevention in the world's high incidence, mainland endemic areas is unjustified-and indeed it could be worse than simply this, since it could delay attempts to approach the problem in other ways.

So, 25 years into the DDS era, we have to take stock over a course of events which have, unfortunately, apparently left many of the early hopes unfulfilled. Some of the questions we now need to ask are difficult and perhaps painful. We still do not really know what the right clinical DDS regimen is, in terms of dose and duration; the main purpose of this paper has been to indicate that we know even less about the effective application of the optimal regime, whatever that turns out to be. What is the right balance between re-calling patients too infrequently and too often, and to what extent may this balance vary from one area or culture to another? What are the right yardsticks for success or failure? What is the role of other anti-mycobacterial agents such as rifampicin? For, vastly expensive as rifampicin is, by comparison with DDS, it could be that an increase in clinical effectiveness, together with a decrease in the length of time rifampicin is required, might prove that it is cost-effective. Should we devote increasing attention to studying the advantages and disadvantages of depot preparations? Is large-scale domiciliary treatment really the best use of resources, or would a return to the very close supervision of a smaller number of patients be preferable? Is the integration of leprosy treatment into the health services as a whole really likely to be effective, as well as merely well-intentioned? These are the largely unanswered questions which constitute the "application gap" in leprosy, 
separating what is theoretically effective from its practical implementation, and which exemplify so many of the problems of modern medicine throughout the countries of the world, affluent or poor. Trying to answer them will be challenging and stimulating; in the case of leprosy, increasing attention should be given to the judicious use of the randomized controlled trial in planning the delivery of care (Fox, 1971; Cochrane, 1972), as well as in the more familiar field of assessing antibacterial agents.

The problem of treating leprosy effectively should not, however, distract us from what is in many ways an even more pressing question. Lord Rosenheim's words, quoted earlier in this paper, really identified the "application gap" and the benefits to be derived from bridging it, quite apart from those of acquiring new knowledge. The extract has, however, often been quoted out of the wider context of the passage as a whole, which also pointed out that: "It must increasingly be the purpose of the medical profession, and of all who work with them, to aim at prevention rather than cure", and this, of course, means the acquisition of new knowledge. There is undoubtedly new knowledge of the greatest relevance still to be gathered about leprosy, whether this is to do with the development of a vaccine, with the other factors, besides $M$. leprae itself, which determine clinical onset in those exposed, or with the social and economic changes which have been associated with eradication in previously endemic areas. The preventive approach is, after all, the one we need to pursue as hard as we can in order eventually to bypass the extremely difficult treatment problems with which this paper has been mainly concerned. (An almost identical argument could be developed around several other diseases.) Leprosy has always been a disease of uncertainties, paradoxes and the unexpected. It may be that in the not too disiant future, leprologists thinking about more fundamental issues at the same time as they grapple with the problem of delivering effective care, will come up with new knowledge of the kind required to make the prevention rather than the treatment of leprosy the more realistic objective.

\section{References}

Cochrane, A. L. (1972). Effectiveness and efficiency. Random reflections on health services. Rock Carling Monograph. London: Nuffield Provincial Hospitals Trust.

Ellard, G. A., Gammon, P. T. and Harris, J. M. (1974). The application of urine tests to monitor the regularity of dapsone self-administration. Lepr. Rev. 45, 224.

Faget, G. H., Pogge, R. C., Johansen, F. A., Dinan, J. F., Prejean, B. M. and Eccles, C. G. (1943). The Promin treatment of leprosy: A progress report. Publ. Hith Rep. (Washington) 58, 1729.

Fox, W. (1971). The scope of the controlled clinical trial, illustrated by studies of pulmonary tuberculosis. Bull. Wld Hlth Org. 45, 559.

Lancet (1976). Editorial. ii, 615.

Low, S. J. M. and Pearson, J. M. H. (1974). Do leprosy patients take dapsone regularly? Lepr. Rev. 45, 218.

Meade, T. W. (1974). "Risk factor" studies in leprosy. WHO/LEP/74.4.

Molesworth, B. D. (1969). LEPRA control project in Malawi. Lepr. Rev. 40, 237.

Pearson, J. M. H., Rees, R. J. W. and Waters, M. F. R. (1975). Sulphone resistance in leprosy. A review of one hundred clinical cases. Lancet ii, 69.

Quagliato, R., Bechelli, L. M. and Marques, R. M. (1970). Bacterial negativity and reactivation (relapse) of lepromatous outpatients under sulphone treatment. Int. J. Lepr. 38, 250.

Rosenheim, M. (1968). Health in the world tomorrow. Lancet ii, 821. 